\title{
A Quantitative Comparison of the Lee-Carter Model under Different Types of Non-Gaussian Innovations
}

\author{
Chou-Wen Wang ${ }^{\mathrm{a}}$, Hong-Chih Huang ${ }^{\mathrm{b}}$ and I-Chien Liu ${ }^{\mathrm{c}}$ \\ ${ }^{a}$ Department of Risk Management and Insurance, National Kaohsiung First University of Science and \\ Technology, Kaohsiung, Taiwan. \\ ${ }^{\mathrm{b}}$ Department of Risk Management and Insurance, Research Fellow of Risk and Insurance Research Center, \\ National Chengchi University, Taipei, Taiwan. \\ ${ }^{c}$ Department of Risk Management and Insurance, National Chengchi University, Taipei, Taiwan.
}

In the classical Lee-Carter model, the mortality indices that are assumed to be a random walk model with drift are normally distributed. However, for the long-term mortality data, the error terms of the Lee-Carter model and the mortality indices have tails thicker than those of a normal distribution and appear to be skewed. This study therefore adopts five nonGaussian distributions - Student's $t$-distribution and its skew extension (i.e., generalised hyperbolic skew Student's $t$-distribution), one finite-activity Lévy model (jump diffusion distribution), and two infinite-activity or pure jump models (variance gamma and normal inverse Gaussian) - to model the error terms of the Lee-Carter model. With mortality data from six countries over the period 1900-2007, both in-sample model selection criteria (e.g., Bayesian information criterion, Kolmogorov-Smirnov test, Anderson-Darling test, Cramér-von-Mises test) and out-of-sample projection errors indicate a preference for modelling the Lee-Carter model with non-Gaussian innovations.

The Geneva Papers (2011) 36, 675-696. doi:10.1057/gpp.2011.20

Keywords: stochastic mortality model; non-Gaussian distributions; mortality jumps

\section{Introduction}

The relatively recent recognition that mortality is a stochastic process means that traditional valuation methods that rely on deterministic mortality models likely contribute to mispricing problems. Suitable mortality models instead use mortality risk management tactics to quantify mortality and longevity risks, and provide a foundation for pricing and reserving.

Among all the stochastic mortality models that are available, the Lee-Carter model, proposed by Lee and Carter in 1992, is perhaps the most popular choice, because of its ease of implementation and acceptable prediction errors, as demonstrated by various empirical studies across countries. Several modifications of the Lee-Carter model have been proposed by Brouhns et al., ${ }^{1}$ Renshaw and Haberman, ${ }^{2}$ and Denuit et al. ${ }^{3}$ and further

\footnotetext{
${ }^{1}$ Brouhns et al. (2002).

2 Renshaw and Haberman (2003).

${ }^{3}$ Denuit et al. (2007).
} 
676

revisited by $\mathrm{Li}$ and Chan, ${ }^{4}$ who attempt to provide a broader interpretation. Cairns et al. ${ }^{5}$ propose a two-factor stochastic mortality model, denoted the CBD model, in which the first factor affects mortality at all ages, whereas the second factor affects mortality at higher ages much more than at lower ages. Furthermore, Cairns et al. ${ }^{6}$ classify and compare eight stochastic mortality models, including extensions of the CBD model, using mortality data from England and Wales and from the United States. Haberman and Renshaw ${ }^{7}$ also extend the Lee-Carter model to forecast age-period-cohort mortality rates and test for robustness. However, short-term catastrophe mortality shocks, such as the influenza pandemic in 1918 or the tsunami in December 2004, may lead to much higher mortality rates. It is crucial to address such mortality jumps in modelling mortality dynamics.

To take mortality jumps into account, Biffis $^{8}$ uses affine jump diffusions and models asset prices and mortality dynamics, in the context of risk analysis and market valuation in life insurance contracts. Luciano and Vigna ${ }^{9}$ find, with Italian mortality data, that introducing a jump component provides a better fit than does a diffusion component for stochastic mortality processes. Cox et al. ${ }^{10}$ combine geometric Brownian motion with a compound Poisson process to model the age-adjusted mortality rates for the United States and United Kingdom, using an evaluation of the first pure mortality security, the Swiss Re Vita bond. In addition, Lin and $\mathrm{Cox}^{11}$ combine Brownian motion with a discrete Markov chain and log-normal jump size distribution to price mortality-based securities in an incomplete market framework. Chen and $\operatorname{Cox}^{12}$ incorporate a jump process into the Lee-Carter model and use it to forecast mortality rates and analyse mortality securitisation. These studies all use diffusion processes with jump components (JD) and finite-activity Lévy processes to describe the dynamics of morality rates.

However, non-normal innovations can be generated by heavy-tailed distributions. An alternative set of distributions thus involves infinite-activity, or pure jump, Lévy processes, such as the normal inverse Gaussian (NIG) distributions that appear repeatedly in financial applications as unconditional return distributions ${ }^{13}$ or the variance gamma (VG) distributions of Madan and Seneta. ${ }^{14}$ Another method relies on Student's $t$-distribution $(\mathrm{T})$ and its skew extensions, such as the generalised hyperbolic skew Student's $t$-distribution (GHST), as described by Prause, ${ }^{15}$ Barndorff-Nielsen and Shephard, ${ }^{16}$ Jones and Faddy, ${ }^{17}$ Mencia and Sentana, ${ }^{18}$

${ }^{4} \mathrm{Li}$ and Chan (2007).

${ }^{5}$ Cairns et al. (2006).

${ }^{6}$ Cairns et al. (2009).

${ }^{7}$ Haberman and Renshaw (2009).

${ }^{8}$ Biffis (2005).

${ }^{9}$ Luciano and Vigna (2005).

${ }^{10}$ Cox et al. (2006).

${ }^{11}$ Lin and Cox (2008).

12 Chen and Cox (2009).

${ }^{13}$ Eberlein and Keller (1995), Prause (1997), Rydberg (1997), Bølviken and Benth (2000), Lillest $\varnothing 1$ (2000).

${ }^{14}$ Madan and Seneta $(1987,1990)$.

15 Prause (1999).

${ }^{16}$ Barndorff-Nielsen and Shephard (2001).

${ }^{17}$ Jones and Faddy (2003).

${ }^{18}$ Mencia and Sentana (2004). 
Demarta and McNeil, ${ }^{19}$ and Aas and Haff. ${ }^{20}$ Therefore, this study aims to examine whether mortality indices can be described by jump models, such as JD, NIG and VG, or by the Student's $t$ family, including the T and GHST distributions.

To the best of our knowledge, Hainaut and Devolder ${ }^{21}$ were the first to apply $\alpha$-stable subordinators (infinite-activity, strictly positive, Lévy processes) to model mortality hazard rates. However, in the Lee-Carter model, the first difference of mortality indices may be negative, to reflect mortality improvements. Giacometti et al. ${ }^{22}$ consider both the error distributions of the Lee-Carter model and its mortality index, using the NIG distribution, to model mortality for different age groups. They observe that the NIG distributional assumption for the residuals of the Lee-Carter model is better than the Gaussian one for some age groups. Therefore, in line with their work, this study incorporates the normal, $t, \mathrm{JD}, \mathrm{NIG}, \mathrm{VG}$ and GHST distributions into the original LeeCarter model, in an attempt to fit and forecast mortality rates. We rely on mortality data from six countries - Finland, France, the Netherlands, Sweden, Switzerland and the United States - from 1900 to 2007. We fit the model to mortality rates from 1900 to 1999 using the normal, $t$, JD, VG, NIG, and GHST distributions, then forecast the development of the mortality curve for the subsequent eight years. According to the Jarque-Bera (JB) test statistics, we must largely reject the assumptions of normality for the residuals of the Lee-Carter model and the mortality indices. The results of the Kolmogorov-Smirnov (KS), Anderson-Darling (AD) and Cramér-von-Mises (CvM) tests provide powerful evidence to support the rationality of using heavy-tailed distributions for the residuals of the Lee-Carter model and the first difference of mortality indices. Finally, according to the mean absolute percentage errors (MAPE) in the mortality projection, our empirical results indicate that the GHST distribution is the most appropriate choice for modelling longterm mortality indices for most countries.

The remainder of this paper is organised as follows. In the next section, we illustrate the Lee-Carter model with $t$, JD, VG, NIG and GHST innovations, as well as provide the dynamics of the mortality indices. The subsequent section empirically tests the goodness of fit of the stochastic mortality models with normal, $t, \mathrm{JD}, \mathrm{VG}, \mathrm{NIG}$, and GHST distributions; it also offers mortality projections. The last section draws some conclusions about our findings.

\section{The Lee-Carter model with heavy-tailed innovations}

In this section, we first review the classical Lee-Carter model, under which the mortality index follows an ARIMA model with normal innovations. Using the mortality data of six countries, we find that all the residuals of the Lee-Carter model and the mortality indices exhibit non-zero skewness and excess kurtosis. Therefore, we use the Lee-Carter model with five non-Gaussian distributions - $t$, JD, VG, NIG and GHST - to model both the residuals and the dynamics of the mortality indices.

\footnotetext{
19 Demarta and McNeil (2005).

${ }^{20}$ Aas and Haff (2006).

${ }^{21}$ Hainaut and Devolder (2008).

22 Giacometti et al. (2009).
} 


\section{The Lee-Carter model}

We analyse the changes in mortality as a function of both age $x$ and time $t$. The mortality forecast relies on the classical Lee-Carter model, namely,

$$
\ln \left(m_{x, t}\right)=\alpha_{x}+\beta_{x} k_{t}+e_{x, t}
$$

where $m_{x, t}$ is the central death rate for age $x$ in calendar year $t$, defined as the span from time $t$ to time $t+1$. This structure is designed to capture age-period effects; $\alpha_{x}$ describes the average pattern of mortality for the age group; $\beta_{x}$ represents the agespecific patterns of mortality change, indicating the sensitivity of the logarithm of the force of mortality at age $x$ to variations in the time index $k_{t} ; k_{t}$ explains the time trend of the general mortality level; and $e_{x, t}$ represents the deviation of the model from the observed log-central death rates, which should be a normal distribution with zero mean and a relatively small variance. ${ }^{23}$

We use approximation to fit the three parameters. According to two constraint conditions, $\sum_{t} k_{t}=0$ and $\sum_{x} \beta_{x}=1, \hat{\alpha}_{x}$ is simply the average value over time of $\ln \left(m_{x, t}\right)$, and $\hat{k}_{t}$ is the sum over various ages of $\ln \left(m_{x, t}\right)-\hat{\alpha}_{x}$. Using $\ln \left(m_{x, t}\right)-\hat{\alpha}_{x}$ as the dependent variable and $\hat{k}_{t}$ as the explanatory variable, we can obtain $\hat{\beta}_{x}$ by using a simple regression model without an intercept parameter. Finally, we re-estimate the $\hat{k}_{t}$ by iteration, using actual number of deaths, population, $\hat{\alpha}_{x}$ and $\hat{\beta}_{x}$, such that the actual number of deaths is close to the estimated number of deaths, and the adjusted $\hat{k}_{t}$ is denoted as $\hat{k}_{t}^{*}$.

To forecast future mortality dynamics, Lee and Carter $^{24}$ assume that $\alpha_{x}$ and $\beta_{x}$ remain constant over time and therefore forecast the dynamics of adjusting the mortality index $k_{t}^{*}$ using an $\operatorname{ARIMA}(0,1,0)$ model, as follows:

$$
k_{t}^{*}-k_{t-1}^{*}=\gamma+\varepsilon_{t},
$$

where $\gamma$ is a drift term, and $\varepsilon_{t}$ is a sequence of independent and identically Gaussian random variables with mean 0 and variance $\sigma^{2}$.

\section{Normality tests for the residuals and mortality indices}

In this subsection, we apply the $\mathrm{JB}^{25}$ test to determine empirically the normality of the mortality data of six countries (Finland, France, Netherlands, Sweden, Switzerland, U.S.) from 1900 to 2007 . The mortality data for the first five countries come from the Human Mortality Database website, ${ }^{26}$ whereas the U.S. data come from the National Center Health Statistics website. ${ }^{27}$

\footnotetext{
${ }^{23}$ Lee (2000).

${ }^{24}$ Lee and Carter (1992).

${ }^{25}$ Jarque and Bera (1980).

${ }^{26}$ www.mortality.org/.

${ }^{27}$ www.cdc.gov/nchs/nvss/mortality_tables.htm. Death rate files: HIST290 and GMWK290R. Death files: HIST290A and GMWK23F.
} 

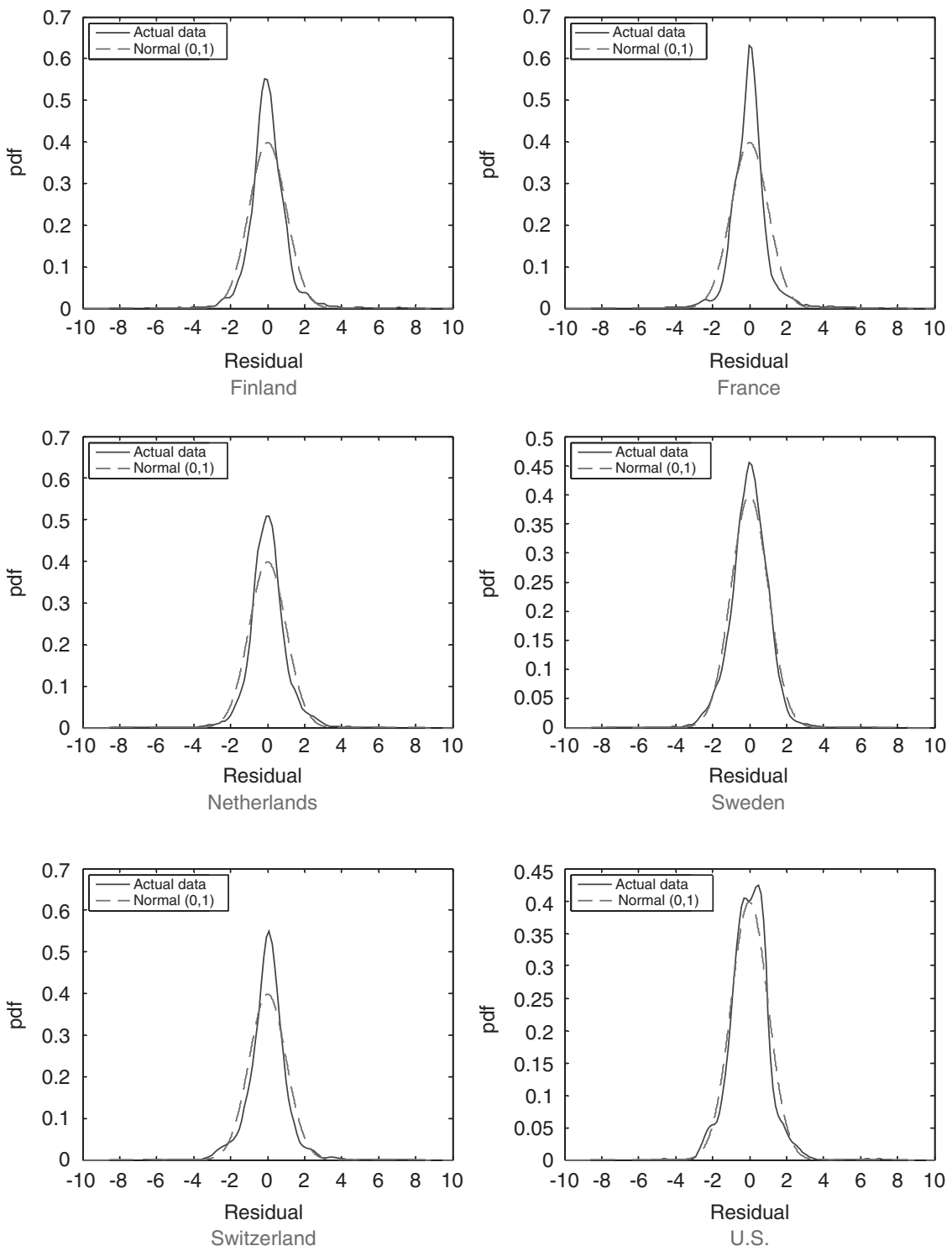

Figure 1. The probability density functions of standardised residuals.

First, we examine the normality test for the residuals in Eq. (1). Figure 1 depicts the probability density function of the standardised residuals. Clearly, the empirical residuals peak around the mean and fatter tails; that is, the residuals are non-normally distributed. Second, Figure 2 reveals the patterns of mortality indices, offering evidence of mortality improvements. We also find a lot of jump points. Chen and $\mathrm{Cox}^{12}$ attribute jump points in the U.S. mortality rate to influenza epidemics, and argue against the naïve belief that a pandemic is a one-time event that cannot happen 

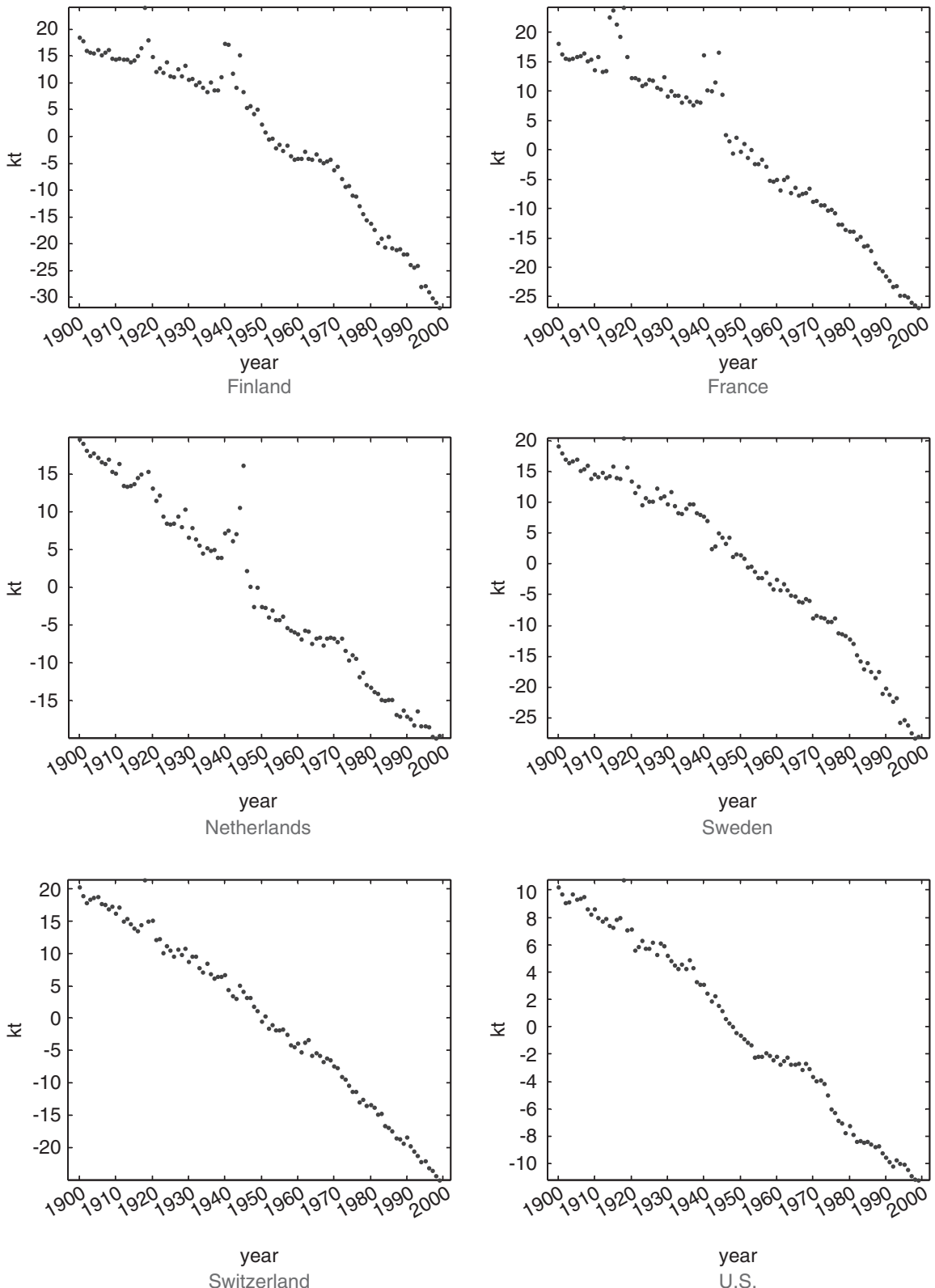

Figure 2. The pattern of mortality indices.

again. We thus cannot just ignore such extreme events. In addition, as we show in Figure 3, the probability density functions of the first differences in the mortality indices exhibit higher central peaks and larger tails than does a normal distribution. Therefore, we can fit the mortality indices to the non-Gaussian distributions. 


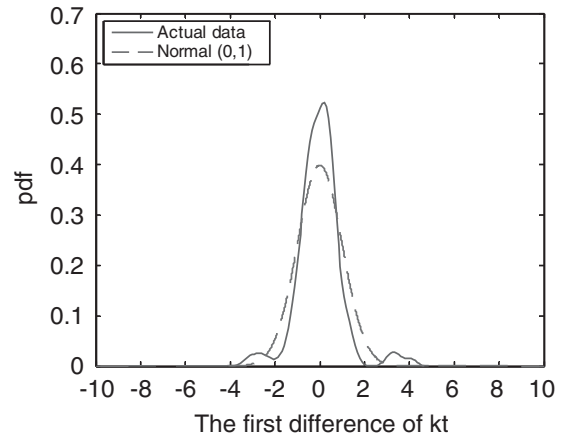

Finland
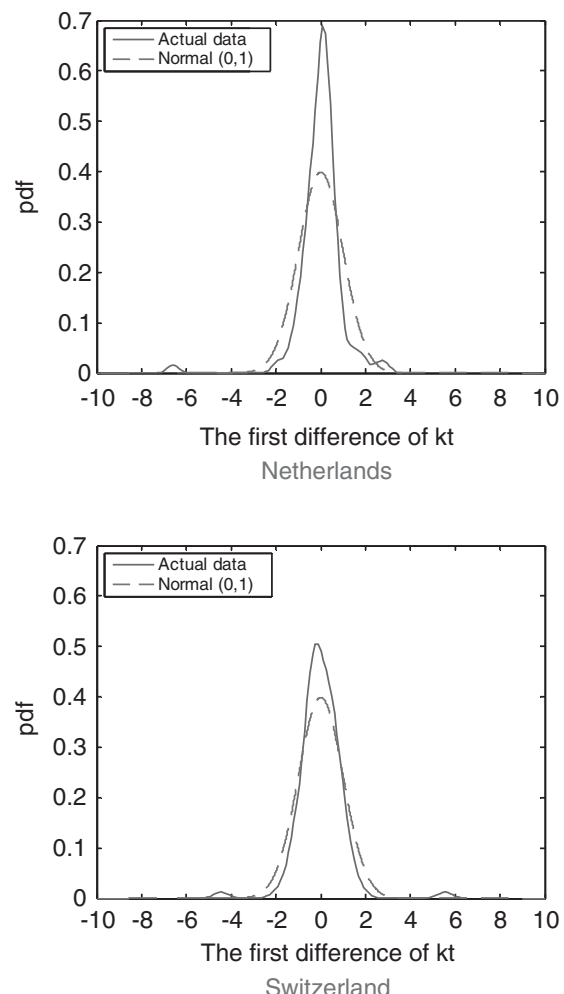

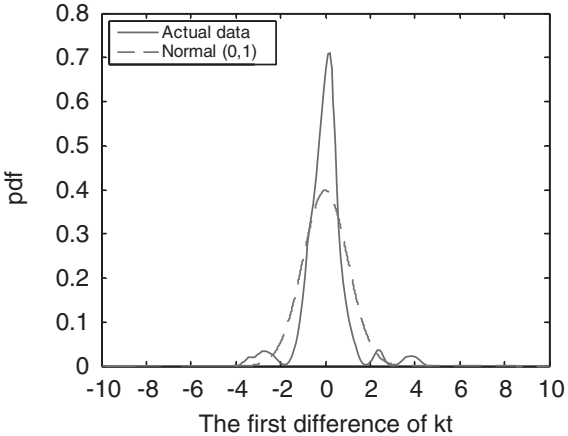

France
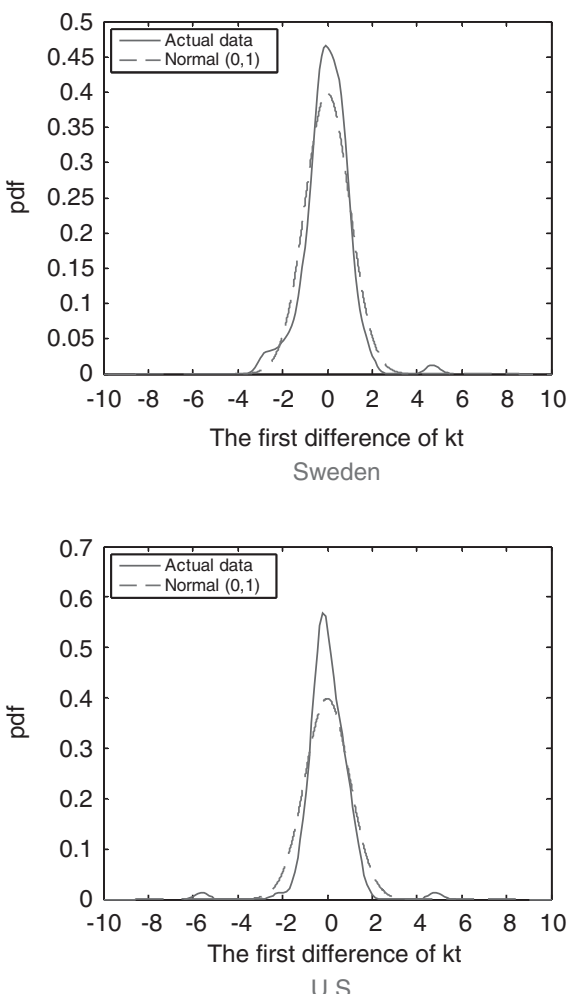

Figure 3. The probability density functions of the first difference in mortality indices.

To examine the assumption of normality of mortality rates in the Lee-Carter model, we also use the JB test statistic, a goodness-of-fit measure of the departure from normality:

$$
J B=n\left[\frac{s^{2}}{6}+\frac{(k-3)^{2}}{24}\right]
$$


Table 1 Skewness, excess Kurtosis and the Jarque-Bera test

\begin{tabular}{|c|c|c|c|c|c|c|}
\hline Country & Finland & France & Netherlands & Sweden & Switzerland & U.S. \\
\hline \multicolumn{7}{|c|}{ Panel A: The residuals of the Lee-Carter model ${ }^{\mathrm{a}}$} \\
\hline Skewness & $\begin{array}{c}0.394 \\
(0.053)\end{array}$ & $\begin{array}{c}0.950 \\
(0.053)\end{array}$ & $\begin{array}{r}-0.298 \\
(0.053)\end{array}$ & $\begin{array}{c}-0.100 \\
(0.053)\end{array}$ & $\begin{array}{c}0.273 \\
(0.053)\end{array}$ & $\begin{array}{c}0.444 \\
(0.074)\end{array}$ \\
\hline Excess Kurtosis & $\begin{array}{c}6.813 \\
(0.107)\end{array}$ & $\begin{array}{c}7.168 \\
(0.107)\end{array}$ & $\begin{array}{c}6.058 \\
(0.107)\end{array}$ & $\begin{array}{c}3.071 \\
(0.107)\end{array}$ & $\begin{array}{c}4.925 \\
(0.107)\end{array}$ & $\begin{array}{c}4.018 \\
(0.148)\end{array}$ \\
\hline JB test & $\begin{array}{c}4,116 \\
{[<0.001]}\end{array}$ & $\begin{array}{c}4,811 \\
{[<0.001]}\end{array}$ & $\begin{array}{c}3,242 \\
{[<0.001]}\end{array}$ & $\begin{array}{c}829 \\
{[<0.001]}\end{array}$ & $\begin{array}{c}2,148 \\
{[<0.001]}\end{array}$ & $\begin{array}{c}776 \\
{[<0.001]}\end{array}$ \\
\hline \multicolumn{7}{|c|}{ Panel B: The first difference in mortality indices ${ }^{\mathrm{b}}$} \\
\hline Skewness & $\begin{array}{c}0.665 \\
(0.246)\end{array}$ & $\begin{array}{c}0.441 \\
(0.246)\end{array}$ & $\begin{array}{c}-2.525 \\
(0.246)\end{array}$ & $\begin{array}{c}0.470 \\
(0.246)\end{array}$ & $\begin{array}{c}0.827 \\
(0.246)\end{array}$ & $\begin{array}{r}-0.661 \\
(0.246)\end{array}$ \\
\hline Excess Kurtosis & $\begin{array}{c}4.439 \\
(0.492)\end{array}$ & $\begin{array}{c}5.339 \\
(0.492)\end{array}$ & $\begin{array}{c}18.396 \\
(0.492)\end{array}$ & $\begin{array}{c}4.601 \\
(0.492)\end{array}$ & $\begin{array}{c}11.446 \\
(0.492)\end{array}$ & $\begin{array}{l}13.476 \\
(0.492)\end{array}$ \\
\hline JB Test & $\begin{array}{c}89 \\
{[<0.001]}\end{array}$ & $\begin{array}{c}121 \\
{[<0.001]}\end{array}$ & $\begin{array}{c}1,501 \\
{[<0.001]}\end{array}$ & {$\left[\begin{array}{c}91 \\
{[<0.001]}\end{array}\right.$} & $\begin{array}{c}552 \\
{[<0.001]}\end{array}$ & $\begin{array}{c}756 \\
{[<0.001]}\end{array}$ \\
\hline
\end{tabular}

${ }^{a}$ The panel presents the skewness and excess kurtosis of the standardised residuals of the Lee-Carter model. Standard errors of the skewness and excess kurtosis given in the parentheses are calculated as $\sqrt{6 / n}$ and $\sqrt{24 / n}$, respectively. $n$ denotes the number of observations. The $p$-values of Jarque-Bera (JB) test are given in brackets.

${ }^{\mathrm{b}}$ The panel presents the skewness and excess kurtosis of the first difference in mortality indices. Standard errors of the skewness and excess kurtosis given in the parentheses are calculated as $\sqrt{6 / n}$ and $\sqrt{24 / n}$, respectively. $n$ denotes the number of observations. The $p$-values of Jarque-Bera (JB) test are given in brackets.

where $n$ is the sample size, $s$ is sample skewness and $k$ is sample kurtosis. Table 1 provides the results of the JB test, together with the skewness and excess kurtosis values, for the residual of the Lee-Carter model and the first difference of the six countries' mortality indices from 1900 to 1999 . The skewness is significantly different from zero, and the excess kurtosis is large. The JB statistics also are significantly large, which means we must reject the assumption of normality. In turn, we use the heavytailed distributions - $t$, JD, VG, NIG and GHST - to model the non-Gaussian property of the error terms in Eqs. (1) and (2).

\section{The Lee-Carter model with non-Gaussian distributions}

Because the residuals of the Lee-Carter model and the mortality indices are nonnormally distributed, we model the error terms, $e_{x, t}$ and $\varepsilon_{t}$, using the five heavy-tailed distributions: $t, \mathrm{JD}, \mathrm{VG}, \mathrm{NIG}$ and GHST.

If a random variable $X$ adheres to a JD distribution, then

$$
X=a+\sigma z+\sum_{i=1}^{N} Y_{i}
$$

where $N$ follows the Poisson distribution with intensity $\lambda ; z$ is a standard normal random variable; and each $Y_{i}$, independent of $z$ and $N$, is a normal distribution 
with mean $\mu$ and variance $\delta^{2}$. Therefore, the probability density function takes the form:

$$
\begin{aligned}
f_{J D}(x \mid a, \sigma, \lambda, \mu, \delta) & =\sum_{n=0}^{\infty} \Phi\left(a+N \mu, \sigma^{2}+N \delta^{2} \mid N=n\right) \operatorname{Prob}(N=n), \\
& =\sum_{n=0}^{\infty} \frac{\lambda^{n} e^{-\lambda}}{n !} \Phi\left(a+n \mu, \sigma^{2}+n \delta^{2}\right),
\end{aligned}
$$

where $\Phi\left(\mu, \sigma^{2}\right)$ is a normal probability density function with mean $\tilde{\mu}$ and variance $\tilde{\sigma}^{2}$.

The $t, \mathrm{VG}, \mathrm{NIG}$ and GHST distributions are the special cases of the generalised hyperbolic $(\mathrm{GH})$ model proposed by Barndorff-Nielsen ${ }^{28}$ and offer flexible tools for modelling the empirical distribution of financial data that exhibit skewness, leptokurtosis and fat tails. ${ }^{29}$ The generalised hyperbolic probability density function is

$$
f_{G H}(x \mid \alpha, \beta, \delta, \gamma, \theta)=\frac{\left(\frac{\sqrt{\alpha^{2}-\beta^{2}}}{\delta}\right)^{\gamma}}{\sqrt{2 \pi}\left(K_{\gamma}\left(\delta \sqrt{\alpha^{2}-\beta^{2}}\right)\right)} e^{\beta(x-\theta)} \frac{K_{\gamma-\frac{1}{2}}\left(\alpha \sqrt{\delta^{2}+(x-\theta)^{2}}\right)}{\left(\frac{\sqrt{\delta^{2}+(x-\theta)^{2}}}{\alpha}\right)^{\frac{1}{2}-\gamma}}
$$

where $K_{\gamma}$ is the modified Bessel function of the second kind with index $\gamma ; \delta$ is the scale parameter; $\theta$ is the shift parameter; and $\gamma, \alpha$ and $\beta$ determine the shape of the $\mathrm{GH}$ distribution. The parameters must fulfil the following constraints:

$$
\begin{array}{ll}
\delta \geqslant, \alpha>|\beta| & \text { if } \gamma>0 . \\
\delta>0, \alpha>|\beta| & \text { if } \gamma=0 . \\
\delta>0, \alpha \geqslant|\beta| & \text { if } \gamma<0 .
\end{array}
$$

When $\gamma=-0.5$, realising that $K_{1 / 2}(x)=\sqrt{\pi / 2} x^{-1 / 2} e^{-x}$ and $K_{-\gamma}(x)=K_{\gamma}(x)$, we obtain the NIG distribution with the following density function:

$$
f_{N I G}(x \mid \alpha, \beta, \delta, \theta)=\frac{\alpha \delta}{\pi} \exp \left(\delta \sqrt{\alpha^{2}-\beta^{2}}+\beta(x-\theta)\right) \frac{K_{1}\left(\alpha \sqrt{\delta^{2}+(x-\theta)^{2}}\right)}{\sqrt{\delta^{2}+(x-\theta)^{2}}}
$$

This distribution is one of the most promising versions of the $\mathrm{GH}$ distribution for asset returns, because it possesses several attractive theoretical properties and

${ }^{28}$ Barndorff-Nielsen (1977, 1978).

${ }^{29}$ In the empirical analyses, we also use the GH distribution to fit the mortality data of our six countries. However, the calibration results of the GH distribution always reduce to those of the GHST distribution, so we focus on this special case instead of the broader GH distribution. 
analytical tractability. It therefore appears frequently in financial applications as an unconditional return distribution ${ }^{13}$ and for stochastic mortality modelling. ${ }^{22}$

If we let $\delta=0$ in Eq. (6), we can obtain the VG distribution, with the following density function:

$$
f_{V G}(x \mid \alpha, \beta, \gamma, \theta)=\frac{\left(\alpha^{2}-\beta^{2}\right)^{\gamma}|x-\theta|^{\gamma-0.5} K_{\gamma-0.5}(\alpha|x-\theta|)}{\sqrt{\pi}(2 \alpha)^{\gamma-0.5} \Gamma(\gamma)} \exp (\beta(x-\theta)) .
$$

Note that when $\alpha=(G+M) / 2, \beta=(G-M) / 2$ and $\gamma=C$, we obtain the VG distribution, which is a special case of the CGMY distribution defined by Carr et al. ${ }^{30}$

If instead we let $\lambda=-v / 2$ and $\alpha \rightarrow|\beta|$ in Eq. (6), and we realise that $K_{\lambda}(x) \sim \Gamma(\lambda) 2^{\lambda-1} x^{-\lambda}$ as $x \rightarrow 0$, we can obtain the density of the GH skew Student's $t$-distribution (GHST), proposed by Aas and Haff, ${ }^{20}$ as follows:

$$
f_{G H S T}(x \mid \beta, v, \delta, \theta)=\frac{\delta^{v}|\beta|^{\frac{v+1}{2}}\left(\sqrt{\delta^{2}+(x-\theta)^{2}}\right)^{-\frac{v+1}{2}} e^{\beta(x-\theta)}}{\sqrt{\pi} 2^{\frac{v-1}{2}} \Gamma(v / 2)} K_{\frac{v+1}{2}}\left(|\beta| \sqrt{\delta^{2}+(x-\theta)^{2}}\right) .
$$

Similarly, letting $\delta=\sqrt{v}$ and $\alpha=\beta=0$ in Eq. (10), we obtain the Student's $t$-distribution with $v$ degrees of freedom. Therefore, the GHST distribution is one of the skew extensions of Student's $t$-distribution.

For mortality data at age $x=1, \ldots, g$ and time period $t=1, \ldots, T$, the calibrated parameters of the Lee-Carter model can be obtained by maximising the sample loglikelihood function (LLF),

$$
L L F=\sum_{x=1}^{g} \sum_{t=1}^{T} \ln \left(f\left(e_{x t} \mid \Theta\right)\right)
$$

with respect to $\Theta$, which satisfies two constraint conditions, $\sum_{t} k_{t}=0$ and $\sum_{x} \beta_{x}=1 .^{31}$ As suggested by Lee and Carter, ${ }^{24}$ we re-estimate the $k_{t}$ factors by iteration, given the values of $\alpha_{x}$ and $\beta_{x}$ we obtained in the maximum likelihood estimation, such that the implied number of deaths equals the actual number of deaths, or

$$
D_{t}=\sum_{x} N_{x, t} \exp \left(\alpha_{x}+\beta_{x} k_{t}\right), \quad t=1, \ldots, T
$$

${ }^{30}$ Carr et al. (2002).

${ }^{31}$ Let $a=-\lambda \mu$ in the JD model and $\theta=-\beta \delta K_{\lambda+1}\left(\delta \sqrt{\alpha^{2}-\beta^{2}}\right) /\left(\sqrt{\alpha^{2}-\beta^{2}} K_{\lambda}\left(\delta \sqrt{\alpha^{2}-\beta^{2}}\right)\right)$ in the special cases of the GH model. Thus, we ensure that the mean of error terms equals 0 . 
where $D_{t}$ is the total number of deaths in year $t$, and $N_{x, t}$ is the total population of age group $x$ at time $t$. Using the re-estimated mortality indices, we can calculate the parameters of Eq. (2) by maximising LLF, as follows:

$$
\sum_{t=1}^{T} \ln \left(f\left(\varepsilon_{t}\right)\right) .
$$

\section{Empirical analysis}

In this section, we illustrate the mortality data and investigate the goodness-of-fit distributions for the residuals of the Lee-Carter model and the first difference of mortality indices. Using the mortality data from 1900 to 1999, we first fit the residuals of the Lee-Carter model with our six distributions: normal, $t, \mathrm{JD}, \mathrm{VG}, \mathrm{NIG}$ and GHST. We then fit the first difference of $k_{t}$ from the best goodness-of-fit model, according to the Bayesian information criterion (BIC), to the same six distributions and project the subsequent eight-year mortality rates.

\section{Model comparison}

For the sake of comparison, we use the LLF, Akaike information criterion (AIC), ${ }^{32}$ $\mathrm{BIC},{ }^{33} \mathrm{KS}$ test, ${ }^{34} \mathrm{AD}$ test, ${ }^{35}$ and $\mathrm{CvM}$ test ${ }^{36}$ as goodness-of-fit measures. The AIC is defined as

$$
A I C=-L L F+N P S,
$$

where NPS is the effective number of parameters being estimated. The BIC is defined as

$$
B I C=-L L F+0.5 \times N P S \times \log (N O S)
$$

where NOS is the number of observations. For these criteria, a higher value of LLF and a smaller value of AIC and BIC indicate a better goodness of fit for the mortality model.

For the KS test, the null hypothesis is $H_{0}: G(x)=F(x ; \Theta)$ for all sample data $x$ and the parameters $\Theta$ of the distribution, where $G(x)$ represents the empirical distribution

\footnotetext{
${ }^{32}$ Akaike (1974).

${ }^{33}$ Schwarz (1978).

34 Kolmogorov (1933).

35 Stephens (1974).

36 Anderson (1962).
} 
function of the sample mortality index and $F(x ; \Theta)$ is the hypothesised cumulative density distribution (CDF). The test statistic is defined as

$$
K S=\sup _{\{x\}}|F(x ; \Theta)-G(x)| .
$$

Thus a higher $p$-value in the KS test means a better goodness of fit for the mortality model.

The AD test is a modification of the KS test, which also determines whether a sample of data comes from a specific distribution. However, unlike the KS test, the AD test focuses on the weight of the tail. Its null hypothesis is that the data follow a specific distribution. The AD test statistic is defined as

$$
A D^{2}=-N O S-S
$$

where

$$
S=\sum_{i=1}^{N O S} \frac{(2 i-1)}{N O S}\left[\ln F\left(y_{i} ; \Theta\right)+\ln \left(1-F\left(y_{N O S+1-i} ; \Theta\right)\right)\right]
$$

$F$ is a cumulative distribution function of the specified distribution; and $y_{i}$ are the observed values in increasing order. A lower value of the test statistic indicates a higher possibility that the mortality data come from the distribution $F$.

The CvM test, an alternative to the KS test, is a criterion used to judge the goodness of fit of a probability distribution, compared with a given empirical distribution function. The test statistic CvM is defined as

$$
C v M=\frac{1}{12 N O S}+\sum_{i=1}^{N O S}\left[\frac{2 i-1}{2 N O S}-F\left(y_{i} ; \Theta\right)\right]^{2}
$$

A lower value of this test statistic indicates a higher possibility that the mortality data come from the distribution $F$. Each test offers some benefits. That is, the KS test is known for the independence of its critical values from the tested distribution. Compared with the KS test, the main advantage of the AD test is that it assigns more weight to the tails of the distribution. Similarly, the CvM test incorporates information about the total sample and is insensitive to a slight dislocation of the empirical CDF. However, a major disadvantage of the CvM and AD tests is that the critical values depend on the analysed distribution. ${ }^{37}$

${ }^{37}$ We obtain the critical values through a Monte Carlo simulation with the estimated parameters (see Chernobai et al., 2007, p. 219). 
Table 2 Goodness-of-fit measures for the residuals of the Lee-Carter model

\begin{tabular}{lcccccc}
\hline Model & LLF & AIC & BIC & LLF rank & AIC rank & BIC \\
\hline Panel A & The & Finland mortality & data & & & \\
Normal & 789.78 & -646.78 & -242.82 & 6 & 6 & \\
$t$ & $1,088.73$ & -944.72 & -537.95 & 4 & 3 & \\
JD & $1,094.23$ & -948.23 & -535.80 & 3 & 5 & 1 \\
VG & $1,073.33$ & -928.33 & -518.73 & 5 & 2 \\
NIG & $1,105.54$ & -960.54 & -550.94 & 1 & 2
\end{tabular}

Panel B: The France mortality data

$\begin{array}{lrrrrrr}\text { Normal } & 826.85 & -683.85 & -279.90 & 6 & 6 & 6 \\ t & 1,279.79 & -1,135.79 & -729.02 & 4 & 4 & 4 \\ \text { JD } & 1,344.31 & -1,198.31 & -785.88 & 1 & 5 & 1 \\ \text { VG } & 1,277.39 & -1,132.39 & -722.78 & 5 & 5 & 5 \\ \text { NIG } & 1,336.77 & -1,191.77 & -782.17 & 2 & 2 & 2 \\ \text { GHST } & 1,296.69 & -1,151.69 & -742.08 & 3 & 3 & 3\end{array}$

Panel C: The Netherlands mortality data

Normal $\quad 1,878.08-1,735.08$

$\begin{array}{lllllll}\text { Normal } & 1,878.08 & -1,735.08 & -1,331.13 & 6 & 6 & 6 \\ t & 2,094.57 & -1,950.57 & -1,543.79 & 5 & 5 & 4 \\ \text { JD } & 2,103.27 & -1,957.27 & -1,544.84 & 2 & 3 & 3 \\ \text { VG } & 2,102.66 & -1,957.66 & -1,548.06 & 3 & 2 & 2 \\ \text { NIG } & 2,118.45 & -1,973.45 & -1,563.85 & 1 & 1 & 1 \\ \text { GHST } & 2,095.81 & -1,950.81 & -1,541.21 & 4 & 4 & 5\end{array}$

Panel D: The Sweden mortality data

$\begin{array}{lllllll}\text { Normal } & 1,856.17 & -1,713.17 & -1,309.21 & 6 & 6 & 6 \\ t & 1,918.32 & -1,774.32 & -1,367.54 & 5 & 5 & 2 \\ \text { JD } & 1,920.62 & -1,774.64 & -1,362.22 & 2 & 4 & 5 \\ \text { VG } & 1,922.51 & -1,777.51 & -1,367.91 & 1 & 1 & 1 \\ \text { NIG } & 1,919.66 & -1,774.66 & -1,365.06 & 4 & 3 & 4 \\ \text { GHST } & 1,920.00 & -1,775.00 & -1,365.40 & 3 & 2 & 3\end{array}$

Panel E: The Switzerland mortality data

$\begin{array}{lllllll}\text { Normal } & 1,612.69 & -1,469.69 & -1,065.74 & 6 & 6 & 6 \\ t & 1,806.21 & -1,662.21 & -1,255.43 & 5 & 4 & 4 \\ \text { JD } & 1,815.79 & -1,669.79 & -1,257.36 & 3 & 2 & 3 \\ \text { VG } & 1,826.48 & -1,681.48 & -1,271.88 & 2 & 2 & 2 \\ \text { NIG } & 1,843.55 & -1,698.55 & -1,288.95 & 1 & 1 & 1 \\ \text { GHST } & 1,806.74 & -1,661.74 & -1,252.14 & 4 & 5 & 5\end{array}$

Panel F: The U.S. mortality data

\begin{tabular}{|c|c|c|c|c|c|c|}
\hline Normal & $1,199.95$ & $-1,074.95$ & -756.82 & 6 & 6 & 6 \\
\hline$t$ & $1,273.77$ & $-1,147.77$ & -827.09 & 4 & 3 & 1 \\
\hline JD & $1,277.47$ & $-1,149.47$ & -823.71 & 1 & 1 & 4 \\
\hline VG & $1,271.16$ & $-1,144.16$ & -820.94 & 5 & 5 & 5 \\
\hline NIG & $1,274.83$ & $-1,147.83$ & -824.61 & 2 & 2 & 2 \\
\hline GHST & $1,274.19$ & $-1,147.19$ & -823.97 & 3 & 4 & 3 \\
\hline
\end{tabular}




\section{In-sample goodness of fit}

Using mortality data from Finland, France, the Netherlands, Sweden, Switzerland and the United States, Table 2 provides the LLF, AIC and BIC results, together with their corresponding ranks. All three criteria indicate that the normal distribution is the worst model for all our mortality data. However, the JD model is the best model for the French mortality data; the NIG model is the best for the mortality data of Finland, the Netherlands and Switzerland; and the VG model is the best option for Sweden. For the U.S. mortality data, the JD model offers the best fit according to the LLF and AIC values, but the $t$ model is the best according to the BIC.

In Table 3 we report the results for the KS, AD and CvM tests, together with their critical values for all six countries. For each country, the three test statistics are greater

Table 3 Goodness-of-fit tests for the residuals of the Lee-Carter model

\begin{tabular}{|c|c|c|c|c|c|c|c|c|c|}
\hline \multirow[t]{3}{*}{ Model } & \multicolumn{3}{|c|}{$K S$} & \multicolumn{3}{|c|}{$A D$} & \multicolumn{3}{|c|}{$C v M$} \\
\hline & \multirow[t]{2}{*}{ Statistic } & \multicolumn{2}{|c|}{ Critical value } & \multirow[t]{2}{*}{ Statistic } & \multicolumn{2}{|c|}{ Critical value } & \multirow[t]{2}{*}{ Statistic } & \multicolumn{2}{|c|}{ Critical value } \\
\hline & & $5 \%$ & $1 \%$ & & $5 \%$ & $1 \%$ & & $5 \%$ & $1 \%$ \\
\hline \multicolumn{10}{|c|}{ Panel A: The Finland mortality data } \\
\hline Normal & $0.061 * *$ & 0.029 & 0.035 & $21.681 * *$ & 2.443 & 3.914 & $3.365^{* *}$ & 0.458 & 0.746 \\
\hline$t$ & $0.031^{*}$ & 0.029 & 0.035 & $2.624^{*}$ & 2.486 & 3.868 & 0.423 & 0.463 & 0.737 \\
\hline JD & 0.024 & 0.029 & 0.035 & 1.769 & 2.491 & 3.799 & 0.252 & 0.452 & 0.716 \\
\hline VG & 0.022 & 0.029 & 0.035 & 1.352 & 2.473 & 3.909 & 0.190 & 0.466 & 0.747 \\
\hline NIG & 0.016 & 0.030 & 0.035 & 1.028 & 2.516 & 3.906 & 0.104 & 0.464 & 0.753 \\
\hline GHST & 0.024 & 0.029 & 0.036 & 1.590 & 2.494 & 4.098 & 0.188 & 0.457 & 0.786 \\
\hline \multicolumn{10}{|c|}{ Panel B: The France mortality data } \\
\hline Normal & $0.094 * *$ & 0.029 & 0.035 & $35.657 * *$ & 2.443 & 3.914 & $5.671 * *$ & 0.458 & 0.746 \\
\hline$t$ & $0.041 * *$ & 0.029 & 0.035 & $7.765^{* *}$ & 2.516 & 3.829 & $0.970 * *$ & 0.463 & 0.724 \\
\hline JD & 0.021 & 0.029 & 0.035 & 1.329 & 2.465 & 3.780 & 0.176 & 0.456 & 0.716 \\
\hline VG & $0.040 * *$ & 0.029 & 0.036 & $4.513^{* *}$ & 2.467 & 3.852 & $0.462^{*}$ & 0.459 & 0.727 \\
\hline NIG & 0.026 & 0.029 & 0.035 & 2.420 & 2.511 & 3.951 & 0.275 & 0.461 & 0.754 \\
\hline GHST & $0.031^{*}$ & 0.029 & 0.035 & $4.020^{*}$ & 2.511 & 4.026 & 0.425 & 0.456 & 0.772 \\
\hline \multicolumn{10}{|c|}{ Panel C: The Netherlands mortality data } \\
\hline Normal & $0.062 * *$ & 0.029 & 0.035 & $18.743 * *$ & 2.443 & 3.914 & $3.118^{* *}$ & 0.458 & 0.746 \\
\hline$t$ & 0.023 & 0.029 & 0.035 & 1.705 & 2.533 & 3.807 & 0.210 & 0.466 & 0.726 \\
\hline JD & 0.018 & 0.029 & 0.035 & 0.858 & 2.473 & 3.762 & 0.130 & 0.458 & 0.710 \\
\hline VG & 0.024 & 0.029 & 0.035 & 2.336 & 2.448 & 3.742 & 0.272 & 0.454 & 0.727 \\
\hline NIG & 0.013 & 0.030 & 0.035 & 0.335 & 2.547 & 3.899 & 0.038 & 0.470 & 0.741 \\
\hline GHST & 0.019 & 0.029 & 0.035 & 1.692 & 2.483 & 3.897 & 0.194 & 0.460 & 0.758 \\
\hline \multicolumn{10}{|c|}{ Panel D: The Sweden mortality data } \\
\hline Normal & $0.031 *$ & 0.029 & 0.035 & $3.721^{*}$ & 2.443 & 3.914 & $0.590^{*}$ & 0.458 & 0.746 \\
\hline$t$ & 0.015 & 0.029 & 0.035 & 0.571 & 2.488 & 3.881 & 0.084 & 0.466 & 0.751 \\
\hline $\mathrm{JD}$ & 0.014 & 0.029 & 0.035 & 0.429 & 2.472 & 3.857 & 0.060 & 0.452 & 0.736 \\
\hline VG & 0.024 & 0.029 & 0.035 & 1.805 & 2.477 & 3.909 & 0.282 & 0.466 & 0.747 \\
\hline NIG & 0.014 & 0.029 & 0.035 & 0.379 & 2.495 & 3.893 & 0.047 & 0.461 & 0.740 \\
\hline GHST & 0.013 & 0.029 & 0.035 & 0.370 & 2.488 & 3.879 & 0.043 & 0.468 & 0.732 \\
\hline
\end{tabular}


Table 3 (continued)

\begin{tabular}{|c|c|c|c|c|c|c|c|c|c|}
\hline \multirow[t]{3}{*}{ Model } & \multicolumn{3}{|c|}{$K S$} & \multicolumn{3}{|c|}{$A D$} & \multicolumn{3}{|c|}{$C v M$} \\
\hline & \multirow[t]{2}{*}{ Statistic } & \multicolumn{2}{|c|}{ Critical value } & \multirow[t]{2}{*}{ Statistic } & \multicolumn{2}{|c|}{ Critical value } & \multirow[t]{2}{*}{ Statistic } & \multicolumn{2}{|c|}{ Critical value } \\
\hline & & $5 \%$ & $1 \%$ & & $5 \%$ & $1 \%$ & & $5 \%$ & $1 \%$ \\
\hline \multicolumn{10}{|c|}{ Panel E: The Switzerland mortality data } \\
\hline Normal & $0.058 * *$ & 0.029 & 0.035 & $17.921 * *$ & 2.443 & 3.914 & $3.033^{* *}$ & 0.458 & 0.746 \\
\hline$t$ & 0.025 & 0.029 & 0.035 & $2.720^{*}$ & 2.470 & 3.948 & 0.327 & 0.460 & 0.742 \\
\hline JD & 0.027 & 0.029 & 0.035 & $2.621^{*}$ & 2.483 & 3.714 & 0.421 & 0.454 & 0.719 \\
\hline VG & 0.016 & 0.029 & 0.035 & 0.865 & 2.456 & 3.805 & 0.108 & 0.454 & 0.730 \\
\hline NIG & 0.017 & 0.030 & 0.036 & 0.699 & 2.579 & 3.978 & 0.081 & 0.473 & 0.756 \\
\hline GHST & 0.025 & 0.029 & 0.035 & $2.677^{*}$ & 2.514 & 3.885 & 0.323 & 0.464 & 0.746 \\
\hline \multicolumn{10}{|c|}{ Panel F: The U.S. mortality data } \\
\hline Normal & $0.050 * *$ & 0.039 & 0.046 & $7.501 * *$ & 2.491 & 3.902 & $1.229 * *$ & 0.464 & 0.748 \\
\hline$t$ & $0.047 * *$ & 0.039 & 0.047 & 1.711 & 2.551 & 4.045 & 0.296 & 0.473 & 0.763 \\
\hline JD & $0.047 * *$ & 0.039 & 0.047 & 1.346 & 2.459 & 3.933 & 0.234 & 0.461 & 0.757 \\
\hline VG & 0.038 & 0.039 & 0.046 & 2.291 & 2.488 & 3.880 & 0.361 & 0.460 & 0.745 \\
\hline NIG & 0.038 & 0.039 & 0.046 & 1.430 & 2.479 & 4.001 & 0.246 & 0.455 & 0.756 \\
\hline GHST & $0.039 *$ & 0.039 & 0.046 & 1.523 & 2.541 & 3.868 & 0.249 & 0.474 & 0.744 \\
\hline
\end{tabular}

$*$ and $* *$ denote significance at the $5 \%$ and $1 \%$ level, respectively.

than the 5 per cent critical value, so the empirical distribution of the residuals does not follow a normal distribution. For the Netherlands and Sweden, no test results reject the null hypothesis that the residuals come from non-Gaussian distributions. In addition, except for the U.S. mortality data, the results of the three tests support the null hypothesis that the residuals come from the best BIC models. For the U.S. mortality data though, the KS statistic rejects the $t$ model, which is the best model according to the $\mathrm{BIC}$, at a 1 per cent significance level. Thus the difference between the theoretical and empirical CDF appears significant. However, if we ignore the dislocation of the empirical CDF, the $t$ model offers better goodness of fit for the U.S. residuals, from the standpoint of the AD and CvM tests. Because the AD and CvM test results do not reject the claim that the error terms in Eq. (1) come from the best models, according to the BIC, we use the mortality indices obtained from the best BIC model to investigate the pattern of innovations in Eq. (2).

Table 4 contains the results for the LLF, AIC and BIC and their corresponding ranks in terms of the normal, $t, \mathrm{JD}, \mathrm{VG}, \mathrm{NIG}$, and GHST distributions for the first difference of mortality indices. The Gaussian model is the worst, according to the LLF, AIC and BIC. The LLF criterion also indicates that the best goodness of fit derives from the NIG model for the Netherlands but from the JD model for the five other countries. Because it introduces a penalty term for the effective number of parameters, the best in-sample goodness of fit changes for the $t$ distribution, except for France and the Netherlands. According to the BIC, the NIG model again is the best fit for the Netherlands, the JD model is the best for France and the $t$ model is the best one for Finland, Sweden, Switzerland and the United States. Table 5 lists the results for the $\mathrm{KS}, \mathrm{AD}$ and CvM tests, together with their critical values, pertaining to the error 
The Geneva Papers on Risk and Insurance-Issues and Practice

690

Table 4 Goodness-of-fit tests for the first difference in mortality indices

\begin{tabular}{|c|c|c|c|c|c|c|}
\hline Model & $L L F$ & $A I C$ & $B I C$ & LLF rank & AIC rank & $B I C r$ \\
\hline \multicolumn{7}{|c|}{ Panel A: The Finland mortality index } \\
\hline Normal & -208.78 & 210.78 & 213.38 & 6 & 6 & 6 \\
\hline$t$ & -195.83 & 198.83 & 202.72 & 4 & 2 & 1 \\
\hline $\mathrm{JD}$ & -192.28 & 197.28 & 203.77 & 1 & 1 & 2 \\
\hline VG & -197.68 & 201.68 & 206.87 & 5 & 5 & 5 \\
\hline NIG & -195.34 & 199.34 & 204.53 & 2 & 3 & 3 \\
\hline GHST & -195.63 & 199.63 & 204.82 & 3 & 4 & 4 \\
\hline
\end{tabular}

Panel B: The France mortality index

$\begin{array}{llll}\text { Normal } & -222.77 & 224.77 & 227.36 \\ t & -204.48 & 207.48 & 211.38 \\ \text { JD } & -195.99 & 200.99 & 207.48 \\ \text { VG } & -204.51 & 208.51 & 213.70 \\ \text { NIG } & -198.66 & 202.66 & 207.85 \\ \text { GHST } & -202.81 & 206.81 & 212.01\end{array}$

$\begin{array}{lll}6 & 6 & 6 \\ 4 & 4 & 3 \\ 1 & 1 & 1 \\ 5 & 5 & 5 \\ 2 & 2 & 2 \\ 3 & 3 & 4\end{array}$

Panel C: The Netherlands mortality index

$\begin{array}{llll}\text { Normal } & -209.29 & 211.29 & 213.88 \\ t & -184.02 & 187.02 & 190.91 \\ \text { JD } & -181.28 & 186.28 & 192.77 \\ \text { VG } & -181.98 & 185.98 & 191.17 \\ \text { NIG } & -179.93 & 183.93 & 189.12 \\ \text { GHST } & -181.40 & 185.40 & 190.59\end{array}$

$\begin{array}{llll}213.88 & 6 & 6 & 6\end{array}$

$\begin{array}{llll}90.91 & 5 & 5 & 3\end{array}$

$\begin{array}{llll}92.77 & 2 & 4 & 5\end{array}$

$\begin{array}{llll}91.17 & 4 & 3 & 4\end{array}$

$\begin{array}{llll}89.12 & 1 & 1 & 1\end{array}$

Panel D: The Sweden mortality index

$\begin{array}{lllllll}\text { Normal } & -183.24 & 185.24 & 187.84 & 6 & 6 & 6 \\ t & -175.64 & 178.64 & 182.53 & 3 & 1 & 1 \\ \text { JD } & -174.79 & 179.79 & 186.28 & 1 & 4 & 5 \\ \text { VG } & -176.59 & 180.59 & 185.78 & 5 & 5 & 4 \\ \text { NIG } & -175.65 & 179.65 & 184.84 & 4 & 3 & 3 \\ \text { GHST } & -175.49 & 179.49 & 184.68 & 2 & 2 & 2\end{array}$

Panel E: The Switzerland mortality index

$\begin{array}{lllllll}\text { Normal } & -168.78 & 170.78 & 173.37 & 6 & 6 & 6 \\ t & -150.54 & 153.54 & 157.43 & 3 & 2 & 1 \\ \text { JD } & -147.09 & 152.09 & 158.58 & 1 & 5 & 2 \\ \text { VG } & -153.58 & 157.58 & 162.77 & 5 & 5 & 5 \\ \text { NIG } & -151.70 & 155.70 & 160.89 & 4 & 4 & 4 \\ \text { GHST } & -150.37 & 154.37 & 159.56 & 2 & 3 & 3\end{array}$

Panel F: The U.S. mortality index

\begin{tabular}{|c|c|c|c|c|c|c|}
\hline Normal & -92.74 & 94.74 & 97.34 & 6 & 6 & 6 \\
\hline$t$ & -69.39 & 72.39 & 76.28 & 3 & 2 & 1 \\
\hline JD & -65.90 & 70.90 & 77.38 & 1 & 1 & 2 \\
\hline VG & -70.89 & 74.89 & 80.08 & 5 & 5 & 5 \\
\hline NIG & -70.73 & 74.73 & 79.92 & 4 & 4 & 4 \\
\hline GHST & -69.35 & 73.35 & 78.54 & 2 & 3 & 3 \\
\hline
\end{tabular}


Chou-Wen Wang et al. A Quantitative Comparison of the Lee-Carter Model

Table 5 Goodness-of-fit tests for the first difference in mortality indices

\begin{tabular}{|c|c|c|c|c|c|c|c|c|c|}
\hline \multirow[t]{3}{*}{ Model } & \multicolumn{3}{|c|}{$K S$} & \multicolumn{3}{|c|}{$A D$} & \multicolumn{3}{|c|}{$C v M$} \\
\hline & \multirow[t]{2}{*}{ Statistic } & \multicolumn{2}{|c|}{ Critical value } & \multirow[t]{2}{*}{ Statistic } & \multicolumn{2}{|c|}{ Critical value } & \multirow[t]{2}{*}{ Statistic } & \multicolumn{2}{|c|}{ Critical value } \\
\hline & & $5 \%$ & $1 \%$ & & $5 \%$ & $1 \%$ & & $5 \%$ & $1 \%$ \\
\hline \multicolumn{10}{|c|}{ Panel A: The Finland mortality index } \\
\hline Normal & 0.122 & 0.130 & 0.158 & 2.351 & 2.477 & 3.933 & 0.329 & 0.461 & 0.737 \\
\hline$t$ & 0.052 & 0.131 & 0.158 & 0.364 & 2.502 & 3.944 & 0.043 & 0.462 & 0.746 \\
\hline JD & 0.053 & 0.130 & 0.159 & 0.218 & 2.529 & 3.941 & 0.037 & 0.457 & 0.753 \\
\hline VG & 0.063 & 0.130 & 0.159 & 0.555 & 2.481 & 3.994 & 0.068 & 0.465 & 0.769 \\
\hline NIG & 0.053 & 0.130 & 0.158 & 0.355 & 2.470 & 3.700 & 0.051 & 0.457 & 0.707 \\
\hline GHST & 0.054 & 0.131 & 0.157 & 0.313 & 2.509 & 3.923 & 0.038 & 0.459 & 0.728 \\
\hline \multicolumn{10}{|c|}{ Panel B: The France mortality index } \\
\hline Normal & $0.156^{*}$ & 0.130 & 0.158 & $4.333^{* *}$ & 2.477 & 3.933 & $0.720^{*}$ & 0.461 & 0.737 \\
\hline$t$ & 0.104 & 0.131 & 0.158 & 1.438 & 2.476 & 3.932 & 0.233 & 0.458 & 0.735 \\
\hline JD & 0.065 & 0.131 & 0.158 & 0.411 & 2.513 & 3.980 & 0.077 & 0.459 & 0.762 \\
\hline VG & 0.106 & 0.131 & 0.158 & 1.275 & 2.532 & 3.961 & 0.224 & 0.469 & 0.768 \\
\hline NIG & 0.064 & 0.130 & 0.157 & 0.458 & 2.484 & 3.845 & 0.073 & 0.457 & 0.724 \\
\hline GHST & 0.077 & 0.131 & 0.157 & 0.754 & 2.509 & 3.923 & 0.093 & 0.459 & 0.728 \\
\hline \multicolumn{10}{|c|}{ Panel C: The Netherlands mortality index } \\
\hline Normal & $0.144^{*}$ & 0.130 & 0.158 & $4.236^{* *}$ & 2.477 & 3.933 & $0.720^{*}$ & 0.461 & 0.737 \\
\hline$t$ & 0.092 & 0.131 & 0.157 & 1.226 & 2.509 & 3.887 & 0.206 & 0.464 & 0.735 \\
\hline JD & 0.069 & 0.131 & 0.159 & 0.361 & 2.551 & 3.899 & 0.070 & 0.469 & 0.760 \\
\hline VG & 0.064 & 0.131 & 0.157 & 0.640 & 2.480 & 3.881 & 0.077 & 0.455 & 0.744 \\
\hline NIG & 0.052 & 0.131 & 0.157 & 0.218 & 2.481 & 3.884 & 0.027 & 0.459 & 0.749 \\
\hline GHST & 0.058 & 0.130 & 0.156 & 0.348 & 2.508 & 3.825 & 0.046 & 0.456 & 0.728 \\
\hline \multicolumn{10}{|c|}{ Panel D: The Sweden mortality index } \\
\hline Normal & 0.093 & 0.130 & 0.158 & 1.192 & 2.477 & 3.933 & 0.164 & 0.461 & 0.737 \\
\hline$t$ & 0.054 & 0.131 & 0.158 & 0.289 & 2.503 & 3.944 & 0.036 & 0.462 & 0.746 \\
\hline JD & 0.062 & 0.131 & 0.158 & 0.215 & 2.493 & 3.935 & 0.034 & 0.469 & 0.739 \\
\hline VG & 0.058 & 0.131 & 0.160 & 0.302 & 2.500 & 3.893 & 0.041 & 0.469 & 0.758 \\
\hline NIG & 0.058 & 0.130 & 0.156 & 0.254 & 2.487 & 3.916 & 0.038 & 0.454 & 0.726 \\
\hline GHST & 0.054 & 0.131 & 0.157 & 0.238 & 2.509 & 3.924 & 0.034 & 0.459 & 0.728 \\
\hline \multicolumn{10}{|c|}{ Panel E: The Switzerland mortality index } \\
\hline Normal & 0.104 & 0.130 & 0.158 & 2.244 & 2.477 & 3.933 & 0.310 & 0.461 & 0.737 \\
\hline$t$ & 0.060 & 0.131 & 0.157 & 0.345 & 2.511 & 3.887 & 0.052 & 0.467 & 0.735 \\
\hline JD & 0.063 & 0.130 & 0.158 & 0.243 & 2.515 & 3.902 & 0.047 & 0.464 & 0.746 \\
\hline VG & 0.066 & 0.131 & 0.157 & 0.552 & 2.521 & 3.940 & 0.073 & 0.465 & 0.761 \\
\hline NIG & 0.064 & 0.131 & 0.157 & 0.411 & 2.443 & 3.830 & 0.061 & 0.457 & 0.733 \\
\hline GHST & 0.061 & 0.130 & 0.156 & 0.323 & 2.507 & 3.825 & 0.055 & 0.456 & 0.728 \\
\hline \multicolumn{10}{|c|}{ Panel F: The U.S. mortality index } \\
\hline Normal & $0.140^{*}$ & 0.130 & 0.158 & $3.123^{*}$ & 2.477 & 3.933 & 0.456 & 0.461 & 0.737 \\
\hline$t$ & 0.077 & 0.131 & 0.157 & 0.508 & 2.508 & 3.887 & 0.083 & 0.466 & 0.733 \\
\hline JD & 0.094 & 0.130 & 0.158 & 0.484 & 2.510 & 4.052 & 0.098 & 0.461 & 0.757 \\
\hline VG & 0.109 & 0.132 & 0.159 & 0.926 & 2.552 & 4.004 & 0.136 & 0.474 & 0.767 \\
\hline NIG & 0.076 & 0.130 & 0.157 & 0.546 & 2.477 & 3.867 & 0.076 & 0.460 & 0.743 \\
\hline GHST & 0.076 & 0.130 & 0.156 & 0.493 & 2.507 & 3.824 & 0.081 & 0.456 & 0.728 \\
\hline
\end{tabular}

$*$ and $* *$ denote significance at the $5 \%$ and $1 \%$ level, respectively. 
692

terms of the mortality indices. The results reject the notion that the error terms of the mortality indices for France, the Netherlands and the United States come from a normal distribution. All three test results confirm that the error terms of the mortality indices come from non-Gaussian distributions. Therefore, the goodness-of-fit tests consistently indicate that non-Gaussian distributions provide better in-sample goodness-of-fit for the error terms of the mortality indices.

\section{Mortality projection}

For out-of-sample performance, we apply the parameters estimated from 1900 to 1999 and obtain the mortality projection with 1,000,000 simulation paths. For each path, we can calculate MAPE as follows:

$$
M A P E=100 \% \times \frac{1}{n} \sum_{i=1}^{n}\left|\frac{A_{i}-F_{i}}{A_{i}}\right|,
$$

where $A_{i}$ is the historical mortality rate and $F_{i}$ is the forecast mortality rate. When we apply the calibrated parameters of the Lee-Carter model with the best BIC goodnessof-fit innovations, we find the impacts on different distributions of the mortality projection for the mean, $90^{\text {th }}$ percentile, and $95^{\text {th }}$ percentile of MAPE from 2000 to 2007, as we show in Table 6. Lower values indicate better predictive power for the

Table 6 Percentile of MAPE of mortality projection

\begin{tabular}{|c|c|c|c|c|c|c|c|}
\hline Model & Mean & $90 \%$ & $95 \%$ & Mean rank & $90 \%$ rank & $95 \%$ rank & Average rank \\
\hline \multicolumn{8}{|c|}{ Panel A: The Finland mortality data (Unit: \%) } \\
\hline NIG-Normal & 8.273 & 10.406 & 11.132 & 2 & 6 & 6 & 4.67 \\
\hline $\mathrm{NIG}-t$ & 8.356 & 10.270 & 11.016 & 6 & 5 & 4 & 5.00 \\
\hline NIG-JD & 8.301 & 10.195 & 11.047 & 5 & 2 & 5 & 4.00 \\
\hline NIG-VG & 8.259 & 10.240 & 10.987 & 1 & 4 & 2 & 2.33 \\
\hline NIG-NIG & 8.282 & 10.226 & 11.011 & 3 & 3 & 3 & 3.00 \\
\hline NIG-GHST & 8.284 & 10.085 & 10.755 & 4 & 1 & 1 & 2.00 \\
\hline \multicolumn{8}{|c|}{ Panel B: The France mortality data (Unit: \%) } \\
\hline JD-Normal & 4.866 & 6.797 & 7.833 & 6 & 4 & 4 & 4.67 \\
\hline $\mathrm{JD}-t$ & 4.772 & 6.562 & 7.692 & 2 & 2 & 3 & 2.33 \\
\hline JD-JD & 4.862 & 6.893 & 8.359 & 5 & 6 & 5 & 5.33 \\
\hline JD-VG & 4.789 & 6.579 & 7.644 & 3 & 3 & 2 & 2.67 \\
\hline JD-NIG & 4.853 & 6.858 & 8.435 & 4 & 5 & 6 & 5.00 \\
\hline JD-GHST & 4.475 & 5.808 & 6.655 & 1 & 1 & 1 & 1.00 \\
\hline \multicolumn{8}{|c|}{ Panel C: The Netherlands mortality data (Unit: \%) } \\
\hline NIG-Normal & 3.696 & 5.289 & 6.116 & 6 & 4 & 3 & 4.33 \\
\hline $\mathrm{NIG}-t$ & 3.548 & 5.003 & 5.926 & 3 & 2 & 2 & 2.33 \\
\hline NIG-JD & 3.588 & 5.334 & 6.573 & 4 & 5 & 6 & 5.00 \\
\hline $\mathrm{NIG}-\mathrm{VG}$ & 3.641 & 5.355 & 6.511 & 5 & 6 & 5 & 5.33 \\
\hline NIG-NIG & 3.516 & 5.041 & 6.199 & 2 & 3 & 4 & 3.00 \\
\hline NIG-GHST & 3.227 & 4.253 & 4.938 & 1 & 1 & 1 & 1.00 \\
\hline
\end{tabular}


Table 6 (continued)

\begin{tabular}{|c|c|c|c|c|c|c|c|}
\hline Model & Mean & $90 \%$ & $95 \%$ & Mean rank & $90 \%$ rank & $95 \%$ rank & Average rank \\
\hline \multicolumn{8}{|c|}{ Panel D: The Sweden mortality data (Unit: \%) } \\
\hline VG-Normal & 8.020 & 9.853 & 10.446 & 4 & 6 & 4 & 4.67 \\
\hline $\mathrm{VG}-t$ & 7.922 & 9.617 & 10.251 & 1 & 1 & 1 & 1.00 \\
\hline VG-JD & 8.024 & 9.829 & 10.574 & 6 & 5 & 6 & 5.67 \\
\hline VG-VG & 8.015 & 9.780 & 10.419 & 3 & 3 & 2 & 2.67 \\
\hline VG-NIG & 8.020 & 9.789 & 10.473 & 5 & 4 & 5 & 4.67 \\
\hline VG-GHST & 8.013 & 9.731 & 10.419 & 2 & 2 & 3 & 2.33 \\
\hline \multicolumn{8}{|c|}{ Panel E: The Switzerland mortality data (Unit: \%) } \\
\hline NIG-Normal & 3.816 & 4.798 & 5.321 & 5 & 6 & 5 & 5.33 \\
\hline $\mathrm{NIG}-t$ & 3.783 & 4.652 & 5.187 & 4 & 4 & 4 & 4.00 \\
\hline NIG-JD & 3.817 & 4.656 & 5.341 & 6 & 5 & 6 & 5.67 \\
\hline $\mathrm{NIG}-\mathrm{VG}$ & 3.763 & 4.601 & 5.046 & 2 & 2 & 2 & 2.00 \\
\hline NIG-NIG & 3.765 & 4.616 & 5.107 & 3 & 3 & 3 & 3.00 \\
\hline NIG-GHST & 3.741 & 4.542 & 5.012 & 1 & 1 & 1 & 1.00 \\
\hline \multicolumn{8}{|c|}{ Panel F: The U.S. mortality data (Unit: \%) } \\
\hline$t-$ Normal & 3.266 & 4.247 & 4.676 & 6 & 6 & 6 & 6.00 \\
\hline$t-t$ & 3.191 & 3.971 & 4.357 & 2 & 2 & 2 & 2.00 \\
\hline$t-\mathrm{JD}$ & 3.244 & 4.039 & 4.668 & 4 & 3 & 5 & 4.00 \\
\hline$t-\mathrm{VG}$ & 3.265 & 4.118 & 4.548 & 5 & 5 & 4 & 4.67 \\
\hline$t-\mathrm{NIG}$ & 3.228 & 4.047 & 4.457 & 3 & 4 & 3 & 3.33 \\
\hline$t-\mathrm{GHST}$ & 3.180 & 3.943 & 4.314 & 1 & 1 & 1 & 1.00 \\
\hline
\end{tabular}

Note: X-Y model means that the error terms in Eqs. (1) and (2) are the X and Y models, respectively.

fitted distribution. According to the average rank of the MAPE criterion, the normal distribution provides poor mortality projection performance; the $t$ and its skew extension GHST provide the best mortality projection for all mortality data. Thus, the Lee-Carter model with non-Gaussian distributions provides a better mortality projection than that obtained from a normal distribution, in terms of the MAPE criterion.

\section{Conclusions}

Recently, many researchers have examined mortality rates and explored different models. Some studies demonstrate that mortality rate improvements also exhibit jump properties. We therefore attempt to incorporate five heavy-tailed distributions - $t$, JD, VG, NIG and GHST - into the Lee-Carter model. Using mortality data from six countries, we apply the BIC and $\mathrm{KS}, \mathrm{AD}$, and $\mathrm{CvM}$ tests and find consistent support for the non-Gaussian residuals of the Lee-Carter model. Specifically, when we calibrate the parameters of the Lee-Carter model, the JD-JD model ${ }^{38}$ is the best one for French mortality data, the NIG-NIG model is best for the Netherlands, the VG- $t$

\footnotetext{
${ }^{38}$ The terminology "X-Y model" refers to the error terms in Eqs. (1) and (2), respectively.
} 
694

model offers the best goodness of fit for Swedish mortality data, the $t-t$ model is best for the U.S. mortality data, and the NIG- $t$ model is the best one for the mortality data from Finland and Switzerland. For forecasting mortality rates, we find that the normal distribution provides weak mortality projection performance, whereas $t$ and its skew extension provide good mortality projections. Therefore, for applications of the Lee-Carter model, the heavy-tailed distributions appear to be the most appropriate choices for modelling long-term mortality data.

\section{References}

Aas, K. and Haff, I.H. (2006) 'The generalized hyperbolic skew student's t-distribution', Journal of Financial Econometrics 4(2): 275-309.

Akaike, H. (1974) 'A new look at the statistical model identification', IEEE Transactions on Automatic Control AC-19: 716-723.

Anderson, T.W. (1962) 'On the distribution of the two-sample Cramér-Von Mises criterion', The Annals of Mathematical Statistics 33(3): 1148-1159.

Barndorff-Nielsen, O.E. (1977) 'Exponentially decreasing distributions for the logarithm of particle size', Proceedings of the Royal Society of London 353: 409-419.

Barndorff-Nielsen, O.E (1978) 'Hyperbolic distributions and distributions on hyperbolae', Scandinavian Journal of Statistics 5(3): 151-157.

Barndorff-Nielsen, O.E. and Shephard, N. (2001) 'Non-Gaussian Ornstein-Uhlenbeck-based models and some of their uses in financial economics', Journal of the Royal Statistical Society B 63: 167-241.

Biffis, E. (2005) 'Affine processes for dynamic mortality and actuarial valuations', Insurance: Mathematics and Economics 37(3): 443-468.

B $\varnothing$ lviken, E. and Benth, F.E. (2000) 'Quantification of Risk in Norwegian Stocks via the Normal Inverse Gaussian Distribution', Proceedings of the AFIA 2000 Colloquium, Troms $\varnothing$, Norway, International Actuarial Association, pp. 87-98.

Brouhns, N., Denuit, M. and Vermunt, J.K. (2002) 'A Poisson log-bilinear regression approach to the construction of projected life tables', Insurance: Mathematics and Economics 31(3): 373-393.

Cairns, A.J.G., Blake, D. and Dowd, K. (2006) 'A two-factor model for stochastic mortality with parameter uncertainty: Theory and calibration', Journal of Risk and Insurance 73(4): 687-718.

Cairns, A.J.G., Blake, D., Dowd, K., Coughlan, G.D., Epstein, D., Ong, A. and Balevich, I. (2009) 'A quantitative comparison of stochastic mortality models using data from England and Wales and the United States', North American Actuarial Journal 13(1): 1-35.

Carr, P., Geman, H., Madan, D.B. and Yor, M. (2002) 'The fine structure of asset returns: An empirical investigation', Journal of Business 75(2): 305-332.

Chen, H. and Cox, S.H. (2009) 'Modeling mortality with jumps: Applications to mortality securitization', Journal of Risk and Insurance 76(3): 727-751.

Chernobai, A.S, Rachev, S.T. and Fabozzi, F.J. (2007) Operational Risk: A Guide to Basel II Capital Requirements, Models, and Analysis, Chichester, Hoboken, NJ: John Wiley \& Sons, Inc.

Cox, S.H., Lin, Y. and Wang, S.S. (2006) 'Multivariate exponential tilting and pricing implications for mortality securitization', Journal of Risk and Insurance 73(4): 719-736.

Demarta, S. and McNeil, A.J. (2005) 'The $t$ copula and related copulas', International Statistical Review 73(1): 111-129.

Denuit, M., Devolder, P. and Goderniaux, A.C. (2007) 'Securitization of longevity risk: Pricing survivor bonds with Wang transform in the Lee-Carter framework', Journal of Risk and Insurance 74(1): 87-113.

Eberlein, E. and Keller, U. (1995) 'Hyperbolic distributions in finance', Bernoulli 1(3): 281-299.

Giacometti, R., Ortobelli, S. and Bertocchi, M.I. (2009) 'Impact of different distributional assumptions in forecasting Italian mortality rates', Investment Management and Financial Innovations 6(3): 186-193.

Haberman, S. and Renshaw, A.E. (2009) 'On age-period-cohort parametric mortality rate projections', Insurance: Mathematics and Economics 45(2): 255-270. 
Hainaut, D. and Devolder, P. (2008) 'Mortality modelling with Lévy processes', Insurance: Mathematics and Economics 42(1): 409-418.

Jarque, C.M. and Bera, A.K. (1980) 'Efficient tests for normality, homoscedasticity and serial independence of regression residuals', Economic Letters 6(3): 255-259.

Jones, M.C. and Faddy, M.J. (2003) 'A skew extension of the $t$ distribution, with applications', Journal of the Royal Statistical Society B 65: 159-174.

Kolmogorov, A.N. (1933) Grundbegriffe der Wahrscheinlichkeitsrechnung, Berlin: Springer, English translation (1950): Foundations of the Theory of Probability. Chelsea, New York.

Lee, R. (2000) 'The Lee-Carter method for forecasting mortality, with various extensions and applications', North American Actuarial Journal 4(1): 80-93.

Lee, R.D. and Carter, L.R. (1992) 'Modeling and forecasting U.S. mortality', Journal of the American Statistical Association 87(419): 659-675.

Li, S.H. and Chan, W.S. (2007) 'The Lee-Carter model for forecasting mortality, revisited', North American Actuarial Journal 11(1): 68-89.

Lillest $\varnothing 1$, J. (2000) 'Risk analysis and the NIG distribution', Journal of Risk 2(4): 41-56.

Lin, Y. and Cox, S.H. (2008) 'Securitization of catastrophe mortality risks', Insurance Mathematics and Economics 42(2): 628-637.

Luciano, E. and Vigna, E. (2005) Non mean reverting affine processes for stochastic mortality, ICER Applied Mathematics Working Paper No. 4, from SSRN: http://ssrn.com/abstract $=724706$.

Madan, D.B. and Seneta, E. (1987) 'Chebyshev polynomial approximations and characteristic function estimation', Journal of the Royal Statistical Society Series B 49(2): 163-169.

Madan, D.B. and Seneta, E. (1990) 'The variance gamma (VG) model for share market returns', The Journal of Business 63(4): 511-524.

Mencia, F.J. and Sentana, E. (2004) Estimation and testing of dynamic models with generalised hyperbolic innovations, CMFI Working Paper 0411, Madrid, Spain.

Prause, K. (1997) 'Modelling financial data using generalized hyperbolic distributions', FDM Preprint 48, University of Freiburg.

Prause, K. (1999) 'The generalized hyperbolic models: Estimation, financial derivatives and risk measurement', PhD Thesis, Mathematics Faculty, University of Freiburg.

Renshaw, A.E. and Haberman, S. (2003) 'Lee-Carter mortality forecasting with age-specific enhancement', Insurance: Mathematics and Economics 33(2): 255-272.

Rydberg, T.H. (1997) 'The normal inverse Gaussian Lévy process: Simulation and approximation', Communications in Statistics: Stochastic Models 13(4): 887-910.

Schwarz, G. (1978) 'Estimating the dimension of a model', Annals of Statistics 6(2): 461-464.

Stephens, M.A. (1974) 'EDF statistics for goodness of fit and some comparisons', Journal of the American Statistical Association 69(347): 730-737.

\section{About the Authors}

Hong-Chih Huang is Professor and Head of the Department of Risk Management and Insurance at National Chengchi University in Taiwan. He received his $\mathrm{PhD}$ at the Department of Actuarial Mathematics and Statistics at Heriot-Watt University in the U.K. His research interests cover asset liability management, asset allocation, longevity risk and pension.

Chou-Wen Wang is Associate Professor at the Department of Risk Management and Insurance at National Kaohsiung First University of Science and Technology in Taiwan. He received his PhD at the Department of Money and Banking at National Chengchi University in Taiwan and his Masters degree at the Department of Finance at National Sun Yat-Sen University in Taiwan. His research interests cover option 
pricing, financial risk management, equity return modelling, credit risk modelling, mortality modelling and securitisation.

I-Chien Liu is currently a PhD candidate at the Department of Risk Management and Insurance at National Chengchi University in Taiwan. His major is actuarial science and his research subjects include embedded option for insurance products, mortality modelling and longevity risk. 